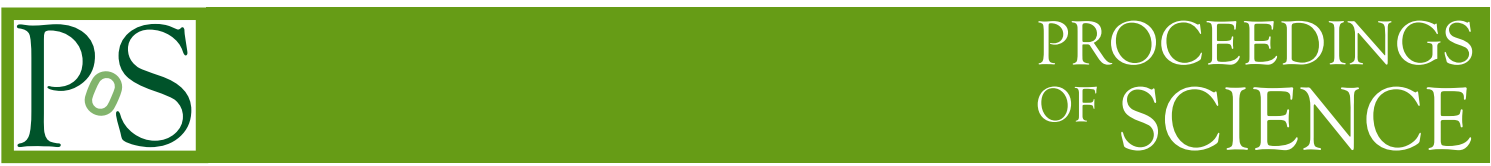

\title{
Very Long Baseline Interferometry with the SKA
}

Zsolt Paragi $^{* 1}$, Leith Godfrey ${ }^{2}$, Cormac Reynolds ${ }^{3}$, Maria Rioja ${ }^{4,5}$, Adam Deller ${ }^{2}$, Bo Zhang $^{6}$, Leonid Gurvits ${ }^{1,37}$, Michael Bietenholz ${ }^{7,38}$, Arpad Szomoru ${ }^{1}$, Hayley Bignall $^{3}$, Paul Boven ${ }^{1}$, Patrick Charlot ${ }^{8}$, Richard Dodson ${ }^{9}, 10$, Sándor Frey ${ }^{11}$, Michael Garrett $^{2,12}$, Hiroshi Imai ${ }^{13}$, Andrei Lobanov ${ }^{14}$, Mark Reid ${ }^{15}$, Eduardo Ros ${ }^{14,39}$, Huib van Langevelde ${ }^{1,12}$, J. Anton Zensus ${ }^{14}$, Xing Wu Zheng ${ }^{16}$, Antxon Alberdi ${ }^{17}$, Iván Agudo $^{1}$, Tao An ${ }^{6}$, Megan Argo ${ }^{18}$, Rob Beswick ${ }^{18}$, Andy D. Biggs ${ }^{19}$, Andreas Brunthaler ${ }^{14}$, Robert M. Campbell ${ }^{1}$, Giuseppe Cimó ${ }^{1}$, Francisco Colomer $^{4}$, Stéphane Corbel $^{20}$, John Conway ${ }^{21}$, David $\mathrm{Cseh}^{22}$, Roger Deane ${ }^{23,33}$, Heino Falcke $^{22}$, Krisztina Gabányi ${ }^{41,11}$, Marcin Gawronski ${ }^{24}$, Michael Gaylard ${ }^{7} ;$ Gabriele Giovannini $^{25}$, Marcello Giroletti ${ }^{25}$, Ciriaco Goddi ${ }^{1}$, Sharmila Goedhart ${ }^{7}$, José L. Gómez $^{17}$, Alastair Gunn ${ }^{18}$, Taehyun Jung ${ }^{9}$, Preeti Kharbb ${ }^{26}$, Hans-Rainer Klöckner ${ }^{14}$, Elmar Körding ${ }^{22}$, Yurii Yu. Kovalev ${ }^{27,14}$, Magdalena Kunert-Bajraszewska ${ }^{24}$, Michael Lindqvist $^{21}$, Matt Lister ${ }^{28}$, Franco Mantovani ${ }^{14,25}$, Iván Martí-Vidal ${ }^{21}$, Mar Mezcua ${ }^{29}$, John McKean ${ }^{2}$, Enno Middelberg ${ }^{30}$, James Miller-Jones ${ }^{3}$, Javier Moldon ${ }^{2}$, Tom Muxlow $^{18}$, Tim O'Brien ${ }^{18}$, Miguel Pérez-Torres ${ }^{17}$, Sergei Pogrebenko ${ }^{1}$, Jonathan Quick $^{7}$, Anthony P. Rushton ${ }^{31,40}$, Richard Schilizzi ${ }^{18}$, Oleg Smirnov ${ }^{32,33}$, Bong Won Sohn ${ }^{9}$, Gabriele Surcis ${ }^{1}$, Greg Taylor ${ }^{34}$, Steven Tingay ${ }^{3}$, Valeriu Tudose ${ }^{35}$, Alexander van der Horst ${ }^{36}$, Joeri van Leeuwen ${ }^{2,36}$, Tiziana Venturi ${ }^{25}$, René Vermeulen $^{2}$, Wouter Vlemmings ${ }^{21}$, Aletha de Witt ${ }^{7}$, Olaf Wucknitz ${ }^{14}$, Jun Yang ${ }^{21}$ ${ }^{1}$ JIVE, ${ }^{2}$ Astron, ${ }^{3}$ ICRAR-Curtin U., ${ }^{4}$ OAN, ${ }^{5}$ ICRAR-UWA, ${ }^{6}$ ShAO,${ }^{7}$ HartRAO, ${ }^{8}$ U. Bordeaux, ${ }^{9}$ KASI, ${ }^{10}$ ICRAR-UWA, ${ }^{11}$ FÖMI SGO, ${ }^{12}$ U. Leiden, ${ }^{13}$ Kagoshima U., ${ }^{14}$ MPIfR,

${ }^{15}$ Harvard-Smithsonian CfA, ${ }^{16}$ SASS-Nanjing U., ${ }^{17}$ IAA-CSIC, ${ }^{18} \mathrm{JBO}$-U. Manchester, ${ }^{19}$ ESO,

${ }^{20}$ CEA Saclay, ${ }^{21}$ OSO,${ }^{22}$ RU Nijmegen, ${ }^{23}$ U. Cape Town, ${ }^{24}$ Torun CfA-Nicolaus Copernicus U.,

${ }^{25}$ IRA-INAF, ${ }^{26}$ IIA Bangalore, ${ }^{27}$ ASC-Lebedev Phys. I., ${ }^{28}$ Purdue U., ${ }^{29}$ IAC, ${ }^{30}$ Ruhr-U. Bochum,

${ }^{31}$ U. Oxford, ${ }^{32}$ Rhodes U., ${ }^{33}$ SKA South Africa, ${ }^{34} U N M,{ }^{35}$ Inst. Sp. Sci., ${ }^{36}$ U. Amsterdam, ${ }^{37}$ TU

Delft, ${ }^{38}$ York U., Toronto, ${ }^{39}$ U. Valencia, ${ }^{40}$ U. Southampton, ${ }^{41}$ U. Szeged

E-mail: zparagi@jive.nl

Adding VLBI capability to the SKA arrays will greatly broaden the science of the SKA, and is feasible within the current specifications. SKA-VLBI can be initially implemented by providing phased-array outputs for SKA1-MID and SKA1-SUR and using these extremely sensitive stations with other radio telescopes, and in SKA2 by realising a distributed configuration providing baselines up to thousands of km, merging it with existing VLBI networks. The motivation for and the possible realization of SKA-VLBI is described in this paper.

Advancing Astrophysics with the Square Kilometre Array

June 8-13, 2014

Giardini Naxos, Italy

*Speaker.

${ }^{\dagger}$ Deceased 


\section{Introduction}

A high angular resolution capability has long been considered an essential part of the Square Kilometre Array (SKA) concept (Garrett 2000; Gurvits 2004; Fomalont \& Reid 2004; Schilizzi et al. 2007; Godfrey et al. 2012). Very long baseline interferometry with the SKA (hereafter SKAVLBI) will provide very sensitive, milliarcsecond (mas) resolution imaging that is important, for example, to study active galactic nuclei (AGN) down to very low luminosities, to understand the detailed physics of jet formation and its coupling to the accretion process, as well as the growth of the first generation of massive black holes in the universe (Agudo et al. 2015), and their role in regulating star formation (a.k.a. feedback processes, see Prandoni \& Seymour 2015; Morganti et al. 2015). The detection of a great number of tidal disruption events (TDE), and radio imaging at mas-scale, will be invaluable for the understanding of jet formation in a pristine environment, and will possibly reveal a new population of massive black holes (MBH, $M_{\mathrm{BH}} \sim 10^{4}-10^{6} M_{\odot}$ ) that may resemble the black hole seeds in the early universe (Donnarumma et al. 2015). Highfidelity SKA-VLBI polarimetric observations will constrain the magnetic field structure close to the jet launch site, providing important constraints on the jet launch mechanism and related processes (Blandford \& Znajek 1977; Blandford \& Payne 1982). In connection with the Cherenkov Telescope Array (CTA), it will help reveal the nature of the large population of hitherto unidentified high-energy sources (Giroletti et al. 2015). The various "exotic" accreting black hole systems that will potentially be revealed by mas-scale deep SKA-VLBI imaging include low-power MBH in the centres of dwarf galaxies (Paragi et al. 2014), off-centre intermediate-mass black holes $\left(M_{\mathrm{BH}} \sim 10^{2}-10^{4} M_{\odot}\right.$, Wolter et al. 2015), and dual- or multiple supermassive black holes ${ }^{1}$ (SMBH, $M_{\mathrm{BH}} \sim 10^{6}-10^{10} M_{\odot}$, Deane et al. 2015) in advanced stages of mergers. It will allow the detection of $10^{6} M_{\odot}$ dark matter haloes from high resolution imaging of gravitationally lensed arcs to investigate galaxy formation scenarios. It will also enable a test of models for dark energy from the measurement of geometric distances to high-redshift galaxies with nuclear water masers (McKean et al. 2015).

Ultra-precise astrometry at the microarcsecond level to determine distances and transverse velocities via the measurement of proper motions and parallaxes of Galactic objects will be possible out to a distance of tens of kpc. Achieving this for a large fraction of the radio pulsar population detected in the SKA Galactic pulsar census will enable strong field tests of gravity in a broad range of relativistic binary systems (Kramer \& Stappers 2015; Shao et al. 2015), the detection of the gravitational wave background (Janssen et al. 2015), tomographic modelling of the large scale Galactic magnetic field and mapping the ionized interstellar plasma in the Galaxy (Han et al. 2015), and constraining the physics of neutron stars (Watts et al. 2015). Curiously, the gravitational wave background might be constrained independently by observing its subtle effect on the apparent position of quasars (e.g. Jaffe 2004, see also Sect. 4.2). Ultra-precise astrometry will be very important for many other classes of compact radio sources with emission in the SKA frequency range - for instance, masers (Green et al. 2015), protostellar objects (e.g. Loinard et al. 2007), and a variety of accreting stellar objects such as novae, isolated black holes, or accreting neutron stars and black holes in binary systems (a.k.a. microquasars, Corbel et al. 2015). Proper motion measurements of stellar-mass black holes will provide constraints on models of their formation,

\footnotetext{
${ }^{1}$ For two recently reported candidates awaiting confirmation see e.g. Gitti et al. (2013); Deane et al. (2014)
} 


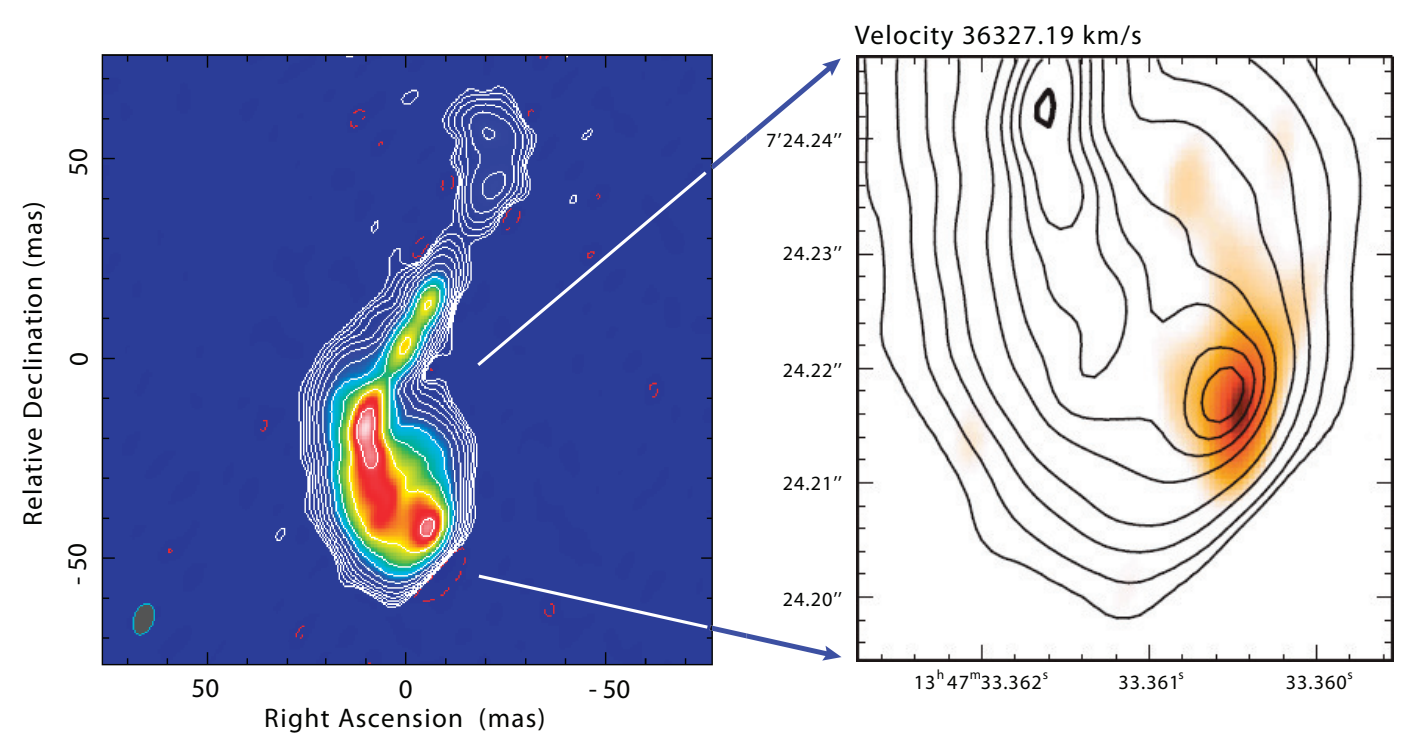

Figure 1: Left: global VLBI continuum image of the young, restarted AGN 4C12.50 at 1.27 GHz. Right: HI absorption (orange-white) superimposed on the continuum image (contours) of the southern lobe. The deepest HI absorption is co-spatial with the termination point of the jet; there is extended HI absorption detected as well. The jet drives a large-scale atomic (HI) and cold molecular (CO) outflow. This result demonstrates that jet-driven outflows may play an important role in AGN feedback mechanisms (Morganti et al. 2013).

while measuring their parallax distances will provide accurate luminosities which are important for tests of accretion physics (e.g. Miller-Jones et al. 2009). For radio-emitting stars, in addition to the distance via parallax, the presence of a planetary companion can be sought via the reflex motion of the star (e.g. Guirado \& Ros 2007; Bower et al. 2009). The excellent sensitivity on very long baselines will mean that extragalactic stellar explosions like supernovae (Bietenholz 2008; PerezTorres et al. 2015) and gamma-ray burst afterglows (GRB, Burlon et al. 2015, but see also Sect. 7) will be imaged, in total intensity as well as in polarization, in more detail than is possible today.

A number of high impact scientific results produced in the last few years demonstrate the potential of very sensitive and flexible SKA-VLBI with mas-scale imaging capability. These include global VLBI HI spectral line imaging of the AGN 4C12.50 (see Fig 1), demonstrating how AGN jets drive large-scale outflows and thus contribute to AGN feedback (Morganti et al. 2013); an accurate parallax measurement of SS Cyg in a series of triggered VLBI experiments (see Fig 3), proving that the disc instability model for accretion is correct (Miller-Jones et al. 2013); and the recent finding that binary orbits may be the driver of $\gamma$-ray emission and mass ejection in classical novae (Chomiuk et al. 2014). To fully explore the broad range of science cases we refer the reader to the various chapters mentioned above, as well as Godfrey et al. $(2011,2012)$. In this paper we focus on practical SKA-VLBI issues and highlight only a few SKA-VLBI science applications.

We describe possible realisations of SKA-VLBI in Sect. 2, along with expected sensitivities based on the SKA1 Baseline Design. Sect. 3 describes calibration requirements and explains the need for forming multiple phased-array beams (in one or more sub-arrays) and why simultaneous production of phased-array and local interferometer data is necessary. Astrometry with SKA-VLBI 


\begin{tabular}{lrrrrr}
\hline \hline SKA Band & $\begin{array}{r}\text { SKA-core } \\
\text { SEFD [Jy }]\end{array}$ & $\begin{array}{r}\text { Bandwidth } \\
{[\mathrm{MHz}]}\end{array}$ & $\begin{array}{r}\text { Remote tel. } \\
\text { SEFD [Jy }]\end{array}$ & $\begin{array}{r}\text { Baseline sens. } \\
60 \mathrm{~s}[\mu \mathrm{Jy}]\end{array}$ & $\begin{array}{r}\text { Image noise } \\
\text { 1hr }[\mu \mathrm{Jy} / \mathrm{beam}]\end{array}$ \\
\hline \hline 50\% SKA1-MID & 5.2 & 256 & 20 & 82 & 9 \\
SKA1-MID & 2.6 & 1024 & 20 & 29 & 3 \\
Full SKA & 0.26 & 2048 & 20 & 3 & 0.05 \\
\hline
\end{tabular}

Table 1: Typical expected $1 \sigma$ baseline and image sensitivities of various SKA-VLBI configurations at $\sim 3-8$ $\mathrm{GHz}$, with the inner $4 \mathrm{~km}$ of SKA core phased up. All the baseline sensitivities are given for a 100m-class remote telescope. 50\% SKA1-MID (early operations): assuming an accompanying array of 5 25-30m dishes and a 100m-class antenna. SKA1-MID - same configuration. Note at $\sim 1-3 \mathrm{GHz}$ and including SKA1-SUR as well will provide a similar sensitivity. Full SKA: 10x more sensitive than SKA1-MID.

will be described in Sect. 4. In Sect. 5 we will show how Gaia astrometry can be improved using SKA-VLBI, and how these complementary facilities may open up new fields of research. This will be followed by introducing the need and potential for SKA-VLBI surveys in Sect. 6. Highlighting the superior resolution of SKA-VLBI even for very faint targets, we will show in Sect. 7 that -in certain cases- it will be possible to resolve extreme GRB afterglows while these are still in the ultra-relativistic regime. Various issues with correlation and data transport will be described in Sect. 8.

\section{VLBI configurations of SKA}

There is a significant difference between the planned operation of the high angular resolution component for SKA1 and SKA2: in SKA1, the SKA core will be operated as a sensitive element (or elements) to be added to existing VLBI networks, increasing the sensitivity but not the resolution of those networks. In this case, the "remote stations" comprise existing facilities, and the SKA will participate as the most sensitive element in an otherwise conventional VLBI array (see Table 1). Key to the success of this approach will be the addition of a few (2-4) remote stations in Africa. Ideal locations for these stations would be the developing African VLBI Network (AVN) stations in Zambia, Ghana, Kenya and Madagascar (Gaylard et al. 2011). These additional stations will provide the short and medium length baselines to the SKA core to give good $u v$-coverage.

The left panel of Figure 2 shows the single-frequency, 12-hours $u v$-coverage that could be obtained at the Galactic centre with a global imaging array including a large number of telescopes. The right panel shows a more typical $u v$-coverage for a 4-hours track on a source at declination $-20^{\circ}$ using just a handful of the more sensitive telescopes available in the global array, but still providing baseline lengths up to $10,000 \mathrm{~km}$ in length. In the coming era of rapidly expanding e-VLBI capabilities the realisation of such an array should not present any significant logistical problems. Coordinated proposals and observations are already offered by the European VLBI Network (EVN), the Chinese VLBI Network (CVN), the Japanese VLBI Network (JVN), the Korean VLBI Network (KVN), the Long Baseline Array (LBA) the Very Long Baseline Array (VLBA), and the High Sensitivity Array (HSA). Table 1 gives the likely sensitivity of arrays that could be formed with SKA1 era assuming various configurations and bandwidths. 

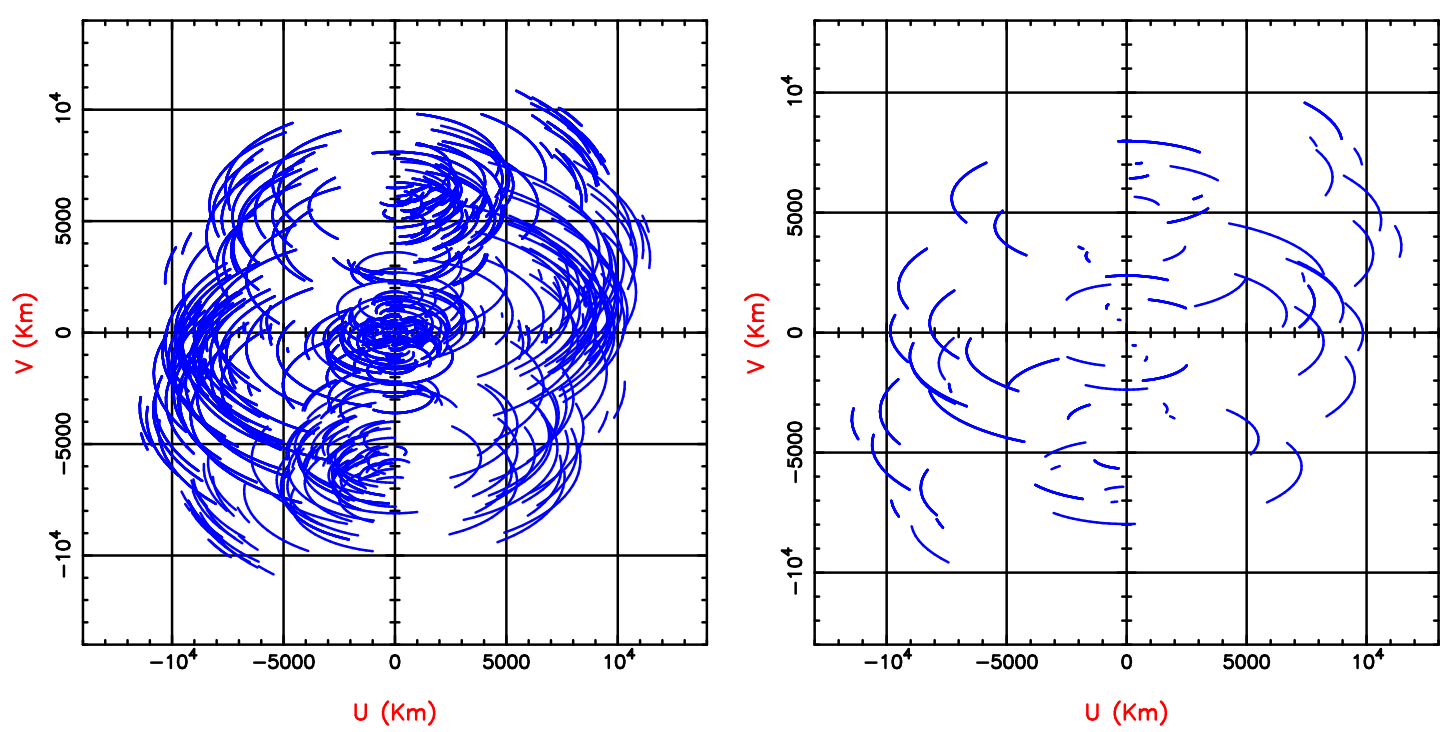

Figure 2: Left: $u v$-coverage of the Galactic Centre in a 12-hours observation with 24 telescopes combined in the EVN, CVN, LBA and the AVN with SKA1-MID. Right: $u v$-coverage of a more typical 4-hours observation of a DEC $-20^{\circ}$ source with 9 telescopes selected from the above configuration.

There are, however, limitations to the approach described above (Garrett 2000). To address these limitations, in SKA2 the remote stations will be an integrated part of the array, with up to $25 \%$ of the collecting area distributed along spiral arms in remote stations, extending thousands of kilometres from the core, and the SKA will effectively operate as a real-time electronic-VLBI (e-VLBI) array. Merging with telescopes from existing VLBI networks will still be possible; note that in this case the SKA processor will have to act as the VLBI correlator (Table 1). Many of the technical requirements for implementation of SKA-VLBI in phase 1 will also be requirements for phase 2, and as such, implementation of the high angular resolution capability in phase 1 will be an essential step to realising the high angular resolution component in phase 2.

Even in the early science phase (50\% SKA1-MID sensitivity) SKA-VLBI will match or exceed the current capabilities of the typical e-EVN configuration ${ }^{2}$, but will be capable of accessing the entire southern sky, including tracking the Galactic center for many hours! SKA-VLBI with the full capacity SKA1-MID and/or SKA1-SUR, especially when adding FAST (Nan et al. 2011), will outperform current global VLBI arrays including the most sensitive telescopes. While we are not considering it further here, we note that VLBI with SKA1-LOW $(v<350 \mathrm{MHz})$ has the potential of using speckles of interstellar scattering to achieve sub-nano-arcsec resolution in certain cases. With current instruments this is possible for some bright pulsars (Pen et al. 2014), but the sensitivity of the SKA is required for more general applications.

\section{SKA-VLBI calibration}

Calibration of the VLBI datastream from the phased-up core of the SKA array would follow

\footnotetext{
${ }^{2}$ The e-EVN consists of EVN telescopes with real-time e-VLBI capability, streaming data at a rate of $1 \mathrm{Gbit} \mathrm{s}^{-1}$. Here we assumed the e-EVN without Arecibo. See http://www.evlbi.org/evlbi/e-vlbi_status.html
} 
much the same methods as currently used for phased arrays in VLBI. When connected element interferometers such as the Australia Telescope Compact Array (ATCA), The Karl G. Jansky Very Large Array (VLA) or the Westerbork Synthesis Radio Telescope (WSRT) are operated as an element of a VLBI network, the internal calibration of the array is used to measure the gains for the individual array antennas. The signals from these are then scaled and summed in a tied array output from the correlator. We assume that the SKA will function along these lines as part of VLBI operations. Besides forming a tied array beam (or rather beams, see below), another crucial requirement is the provision of the metadata, such as the system temperature and weather information, by the array. The gains of the phased-array sum need to be accurately determined and recorded. The metadata will be essential for the accurate conversion of the VLBI correlation into a measure of correlated flux density. These calibrations are particularly important as such data will be dominated by the baselines to the phased SKA because of the large weights these baselines will have due to the large collecting area.

One issue in VLBI is that primary flux density calibrators are not available to further improve on a-priori amplitude calibration. Primary calibrators are resolved on mas scales, while sources that are compact on baselines up to $\sim 10,000 \mathrm{~km}$ are variable. SKA-VLBI will offer a great solution to this by providing local interferometer and phased-array data simultaneously. The flux densities and polarization properties of compact calibrators can be measured using the local interferometer data during the VLBI observations, leading to very accurate flux density and polarization calibration (for both polarization leakage and polarization position angle) of the VLBI data product.

\subsection{Multi-view calibration}

To provide $10 \mu$ as astrometric accuracy using single source phase referencing at $1.6 \mathrm{GHz}$ one would require an extremely nearby reference source. One can estimate from the expressions in Asaki et al. (2007) that the reference sources should be no more than 60 arcsec from the target to provide the required accuracy. The projected source counts suggest that there will not be sufficient calibrator source density to provide this, even at the sensitivities of SKA1-MID to SKA1-SUR baselines (see Table1).

However, using lines of sight to multiple calibrator sources (minimally 3 ) it is possible to solve for a full 2D correction to the spatial atmospheric distortions around the VLBI target, which will provide significantly improved calibration, imaging and astrometric precision compared to that from a single calibrator (see e.g. Rioja et al. 2009, and references therein). This is particularly important in the ionosphere-dominated regime $(<5 \mathrm{GHz})$. The improvement arises from the fitting of a spatial function to the calibration residuals and interpolating this model to the target position, which allows a more accurate calibration solution in the target direction. Simulations show (Jimenez-Monferrer et al. 2010) that by using Multi-view approaches one can achieve an order of magnitude better astrometric accuracy than by using a single calibrator. The necessary calibrator-target separation is $\lesssim 5$ arcminutes, and the probability of detecting suitable calibrators at such distances seems promising at least up to $5 \mathrm{GHz}$ (Godfrey et al. 2011), making this band the best for SKA-VLBI astrometry (described below).

When SKA1-MID and SKA1-SUR are phased for use in SKA-VLBI, the resultant tied-array beams will be narrow ( $\sim 10$ arcseconds at $1.6 \mathrm{GHz}$ if the inner $4 \mathrm{~km}$ of the core is phased up). This is much narrower than the field-of-view of the $25-100 \mathrm{~m}$ class telescopes which will form the 
remainder of the SKA1-VLBI array, and much smaller than the separation between potential SKAVLBI calibrator sources. Accordingly, a different SKA tied array beam will typically be needed per calibrator source. This drives the requirement for a minimum of 4 SKA1-MID and SKA1-SUR VLBI beams.

\section{Astrometry}

\subsection{Differential astrometry}

Differential VLBI astrometry is obtained by carefully calibrating standard phase-referenced VLBI observations (see Reid \& Honma 2014, and references therein). The calibration is derived from one source (or more sources, using Multi-view calibration) registered within the International Celestial Reference Frame (ICRF), and the same local frame is constructed over multiple observations, meaning that systematic contributions to positional errors remain constant to first order. Thus, while the absolute position of the target will be in error by a small but unknown amount, the changes between epochs - including, importantly, annual geometric parallax and source proper motion - are reliable. Compared to absolute astrometry (discussed below), differential astrometry can obtain higher relative precision and can target much fainter objects. Differential astrometry with VLBI provides the highest precision direct distance measurements of objects outside the solar system available in astronomy (see Figure 3), and as such it contributes a crucial rung to the distance ladder.

The attainable accuracy with differential astrometry can be limited by 4 factors: 1) the noiselimited position fit of the target (determined by array resolution and sensitivity and target brightness); 2) the registration of the target within the calibrator frame (limited by the proximity of the calibrator(s) and the calibration solution interval); 3) the stability of the calibrator frame itself (limited by the intrinsic nature of the source(s) used); and, in case of parallax measurements 4) the stability of the target emission centroid. The participation of SKA1-MID and SKA1-SUR in VLBI astrometric observations will lead to considerable reductions in the sum of these error contributions; so much so that the expected noise floor is difficult to extrapolate from present observations.

The instantaneous sensitivity of a VLBI array containing SKA1-MID and SKA1-SUR would be more than a factor of 10 better than the VLBA. This translates into a corresponding reduction in the first error component. Moreover, it means that fainter sources can be used as calibrators. As explained in Sect. 3, several calibrator sources within $\sim 5$ arcminutes could be expected, that will result in up to an order of magnitude reduction in the second error component (which is usually the limiting factor with current astrometric observations). By virtue of the fact that many sources will be used to construct the calibrator frame, cross-checks will be possible allowing the removal of sources which demonstrate discernible structure evolution, mitigating the third error component. This is currently poorly constrained (Fomalont et al. 2011), as it is below the error floor for most current observations. The fourth error component is only relevant for certain targets (such as masers) and can be mitigated by compressing the campaign duration.

Astrometric observations with current instruments are capable of reaching parallax precisions of $\sim 10 \mu$ as (e.g. Deller et al. 2013; Nagayama et al. 2011; Zhang et al. 2013; Reid et al. 2011). SKA-VLBI has the potential to reach parallax accuracies of $3 \mu$ as or better, sufficient for a precise distance to any Galactic object along a line of sight that is not substantially affected by scattering. 

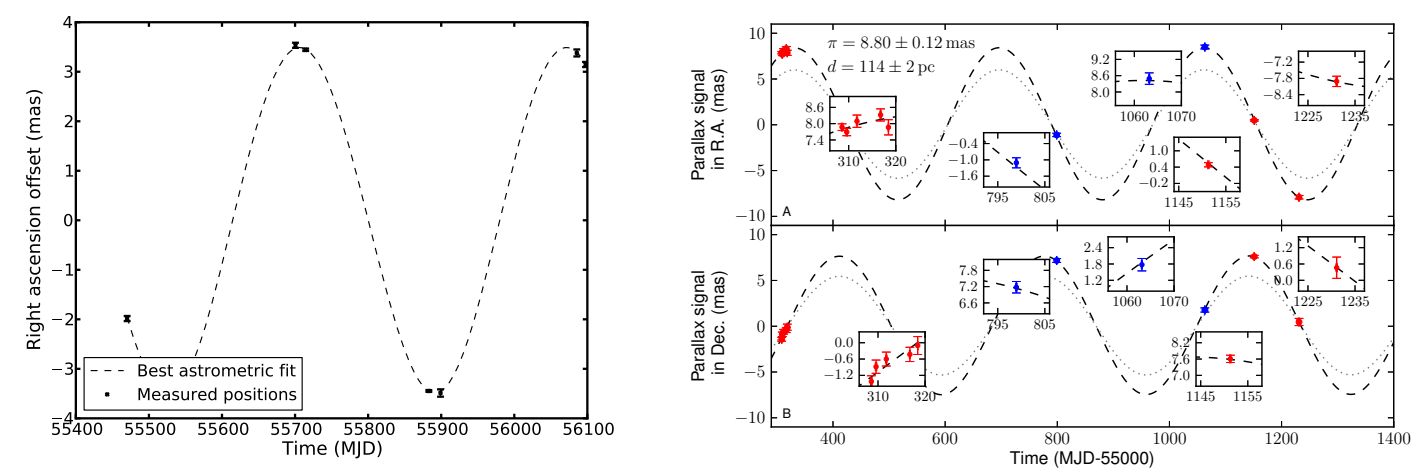

Figure 3: Left: the proper motion and parallax of the binary pulsar J2222-0137 as measured by the VLBA. The distance to this source was measured to $0.4 \%$ accuracy to be 267.3 pc, using 7 observations spread over 20 months (Deller et al. 2013, Fig. 5) Right: VLBA and e-EVN accurate parallax distance measurement of the dwarf nova SS Cyg from a series of triggered VLBI experiments, which removed a major challenge to our understanding of accretion theory (Miller-Jones et al. 2013, Fig. 2)

\subsection{Absolute astrometry and geodesy}

Improving the reference frames - both celestial and terrestrial - that are needed for differential astrometry is the domain of absolute astrometry and geodesy. Absolute astrometry can also be used to study very small secular effects such as the "Galactic aberration" introduced by the acceleration of the Solar System barycentre in the Galactic potential, and apparent quasar proper motions introduced by the low-frequency gravitational wave background (e.g. Gwinn et al. 1997; Titov et al. 2011, and references therein). For these observations, one cannot make use of a nearby calibrator or calibrators to largely eliminate the effect of the ionosphere or troposphere, and so they dominate the error and must be modeled as accurately as possible. As long as the spanned bandwidth is relatively wide, the ionospheric contribution can be precisely measured and compensated because it introduces a dispersive delay. To model the troposphere, observations of many sources at different locations are needed within a short period of time - ideally minutes. The SKA can provide multiple subarrays, but the efficiency will be dictated by the number of non-SKA antennas available to participate.

Unless the number of VLBI-capable antennas in the southern hemisphere is considerably expanded, the addition of SKA1-MID will not result in large improvements in the accuracy of the ICRF. However, SKA2 with intrinsic baselines up to thousands of kilometres as well as higher frequencies, will be able to make a significant improvement over the current ICRF accuracy. To achieve that, it will be necessary to correct for the opacity-shift in AGN jets for the ICRF defining sources (e.g. Paragi, Fejes \& Frey 2000; Porcas 2012, and references therein), which will also be crucial for aligning the radio and the Gaia optical reference frames as explained below.

\section{Synergies with Gaia}

\subsection{Calibrating Gaia parallaxes}

Gaia - the successor to Hipparcos — was launched in the end of 2013, with an ambitious aim to chart a three-dimensional map of the Milky Way. Gaia will use twin telescopes to observe two 
regions in the sky simultaneously to reach the expected parallax accuracy of $\sim 20 \mu$ as at magnitude $\sim 15$. However, even very small periodic variations in the so-called basic angle between the two fields of view could lead to an undesirable global offset of the measured parallaxes (Mignard 2011).

Although the variations are monitored by an on-board metrology system, it is important to verify the Gaia parallaxes by independent methods. Among methods which are in principle available to verify the Gaia parallaxes, distant quasars with practically zero parallaxes are the most promising candidates, but the parallax zero point determined with this method would introduce a possible additional bias from foreground stars contaminating the sample (Windmark et al. 2011). Parallax measurements of stars with accuracy comparable to that of Gaia are possible with VLBI. However, due to the limited sensitivity, only a few stars with optical counterparts observable with Gaia have been measured with VLBI (e.g. Miller-Jones et al. 2013; Ratner et al. 2012). The number of objects detectable with current VLBI arrays is not high enough to calibrate Gaia, therefore we will need SKA-VLBI to accurately measure parallaxes for a significant number of Galactic Gaia targets.

As can be seen in Table 1, the image sensitivity of a moderate SKA-VLBI array is $3 \mu \mathrm{Jy}$ beam $^{-1} \mathrm{hr}^{-0.5}$ at $\sim 3-8 \mathrm{GHz}$. Assuming a baseline length up to $10,000 \mathrm{~km}$ (resolution $\sim 1$ mas), this will ensure a noise-limited position fit with a theoretical precision of a few $\mu$ as for stars with flux density exceeding $\sim 1 \mathrm{mJy}$ (SNR $>100$ ), even if observed in a snapshot mode ( $\sim 10 \mathrm{~min}$. per target). As explained in previous sections, the systematic errors can be reduced significantly by using multiple calibrators within a few arcminutes. For verification of Gaia parallaxes, we need to observe radio targets which have optical counterparts with magnitude $6-15$, of which there are 3699 catalogued $^{3}$. These stars could be first observed in a snapshot survey to measure their radio brightness and compactness; we expect to find hundreds of radio stars as targets for parallax measurements. As a next step, suitable mJy-level calibrators will be sought within a few arcminutes of the targets. All this work can be carried out with 50\% SKA1-MID capabilities in a modest VLBI array (Table 1). It is expected that in phase 1, SKA-VLBI will provide parallaxes with $<10 \mu$ as accuracy for hundreds of stars. To calibrate the Gaia parallax zero point with an uncertainty of 0.6 $\mu$ as, we will need parallax measurements for $\sim 500$ stars.

\subsection{Possible science applications from comparing radio \& optical astrometry}

SKA-VLBI will significantly improve the connection between the celestial reference frames defined in the optical and radio bands. Currently, the most accurate fundamental celestial reference frame is ICRF2, the second realization of the International Celestial Reference Frame (Fey et al. 2009), constructed with dual-frequency $(2.3 / 8.4 \mathrm{GHz})$ VLBI observations of selected radio-loud AGN. By about 2020, Gaia is expected to construct a reference frame with an accuracy similar to or better than that of VLBI, based directly on optical AGN measurements. For maintaining the consistency between the optical and radio frames, it will be essential to align the Gaia and VLBI frames with as many common high-quality reference objects as possible. However, the number of known optically bright AGN detectable with Gaia that also have compact mas-scale radio structures is low (e.g. Bourda et al. 2008). Increasing this number depends largely on having deeper VLBI observations (Bourda et al. 2010), and the high sensitivity SKA-VLBI on long baselines will play an important role here, at least in the southern sky (see Sect. 6).

\footnotetext{
${ }^{3}$ http://www.hs.uni-hamburg.de/EN/For/Kat/radiost.html
} 
Pulsar - white dwarf binary systems provide further opportunity for reference frame ties with Gaia. There are currently $\sim 120$ binary systems known that consist of a white dwarf and a radio pulsar (Manchester et al. 2005). With the SKA pulsar surveys that number could increase 5-fold. Comparison of the positions derived from Gaia and SKA VLBI would considerably enhance the reference frame tie provided by radio stars alone. Importantly, the majority of pulsars with a white dwarf companion are millisecond pulsars, for which $\sim \mu$ as-precision position measurements are possible from timing data alone (Smits et al. 2011). Pulsar timing positions are based on the dynamic solar system frame, while interferometric positions are based on the inertial quasar reference frame therefore pulsars can be used to tie the different reference frames (Bartel et al. 1996). By combining positions derived from pulsar timing data with positions obtained with Gaia and SKA-VLBI, the pulsar-white dwarf binary systems will tie the three reference frames (Gaia frame, the quasar frame and solar system dynamical frame) with better than $10 \mu$ as precision.

To align the radio and optical reference frames using AGN as described above, one has to take into account that the radio and optical peak-brightness positions of compact AGN are not necessarily coincident at the accuracy offered by Gaia and VLBI, a phenomenon known as "core shift". To measure this effect requires observations over a wide range of frequencies, preferably extending to even above $10 \mathrm{GHz}$. With comparable astrometric accuracies in the radio and optical, Gaia will provide important constraints to this by accurately locating the position of the central engine with respect to the observed radio structure. SKA2 will be able to measure core shifts on sub-mas scales for large sample of AGN and thereby constrain AGN jet properties such as the magnetic field strength, the non-thermal particle density, jet power (Lobanov 1998; Kovalev et al. 2008) and even the accretion physics (Zamaninasab et al. 2014). The measurement of the jet power, combined with data at X-ray energies and/or at optical wavelengths, could provide an estimate of the accretion rate and black hole spin (Martínez-Sansigre \& Rawlings et al. 2011).

Comparing Gaia and SKA-VLBI positions of the centres of galaxies will be a great tool for finding astrophysically interesting objects as well. Off-centre compact radio sources could be related to background quasars gravitationally lensed by the galaxy, or indicate dual active nuclei in merging galaxies where one of the components is radio-quiet while the other is optically obscured but radio-loud (e.g. Orosz \& Frey 2013). The latter might be expected for example in minor mergers because the smaller black hole will undergo enhanced accretion episodes during the merger process (Callegari et al. 2011). A particularly interesting case would be the identification of recoiling supermassive black holes that are expelled from the centre of the host galaxy due to three-body interactions (Hoffman \& Loeb 2007), or gravitational-wave recoil after binary coalescence in a galaxy merger (Blecha et al. 2011; Komossa 2012).

\section{SKA-VLBI surveys}

Large field of view (FoV) VLBI surveys have a number of important applications like exploring the AGN content of the universe (identified by their observed high brightness temperature, e.g. Chi, Barthel \& Garrett 2013, see Fig. 4) and revealing the AGN physical properties down to very low accretion rates $\left(10^{-6}-10^{-9} L_{\mathrm{Edd}}\right)$ and masses $\left(<10^{6} M_{\odot}\right)$. Most importantly, combining radio data products with the the "multicolor" sky view of the Large Synoptic Survey Telescope (LSST) will provide invaluable information on a huge number of individual objects; for example, accurate 


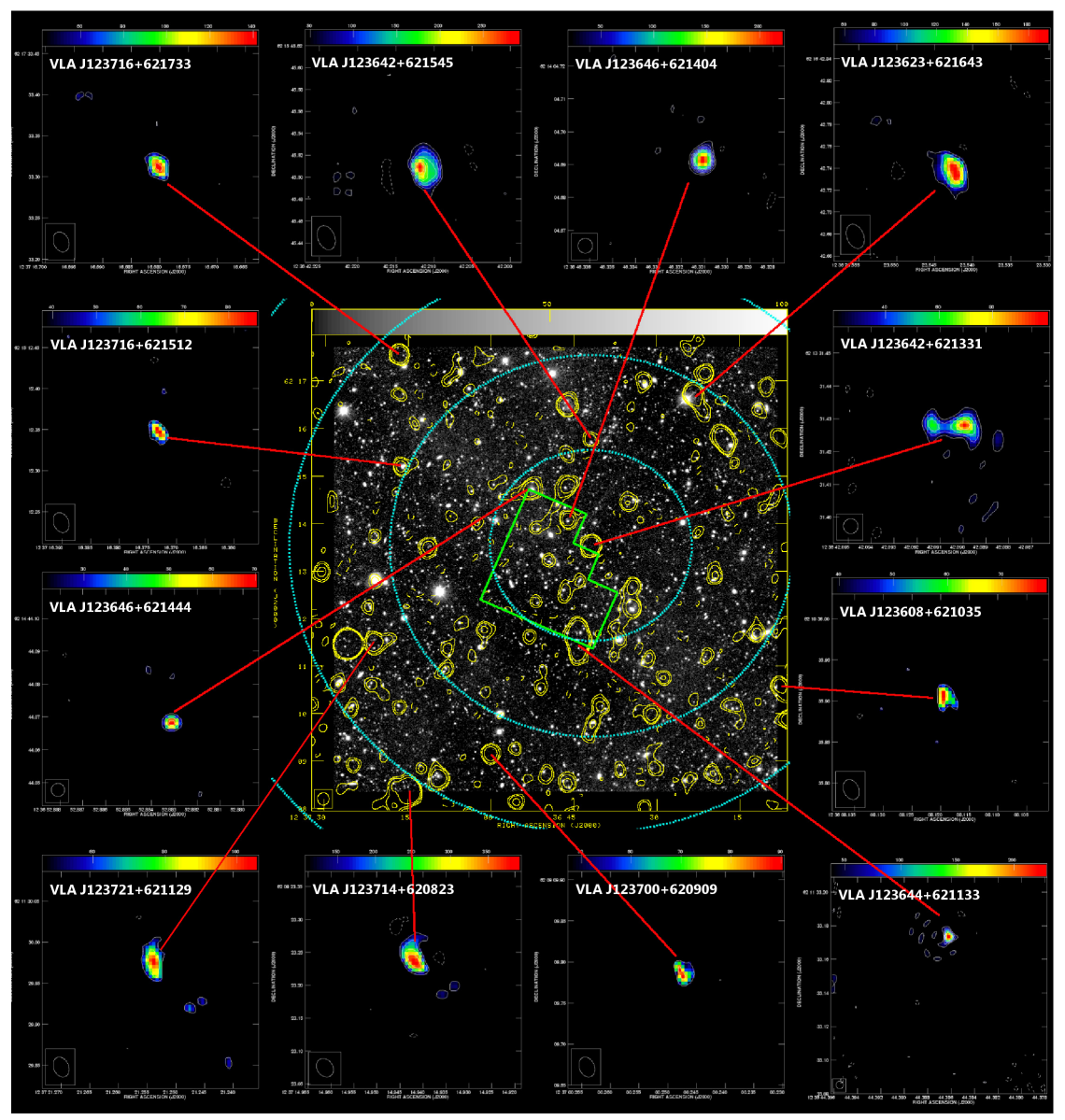

Figure 4: VLBI-detected AGN in the field of Hubble Deep Field North and Flanking Fields (grey scale: optical; yellow contours: WSRT $1.4 \mathrm{GHz}$ ). The cyan circles represent annuli of decreasing resolution and sensitivity, and are drawn at 2, 4, 6, and 8 arcmin radius w.r.t. the phase center which coincides with radio AGN VLA J123642+621331 (Chi, Barthel \& Garrett 2013, Fig. 1).

photometric redshifts will be available for millions of faint targets out to $z>2$, essential to understanding the evolution of activity in the universe as traced by radio emission (Lazio et al. 2014). Although at present, the FoV in VLBI observations has been strongly limited by data averaging in time and frequency, new techniques promise to allow the VLBI imaging of sources over most or all of the primary beam. Wide-field observations to map the full field of view of the primary beam of individual telescopes have been carried out, but this was a computationally challenging exercise (e.g. Lenc et al. 2008). The use of fast hierarchical widefield mapping procedures can reduce the computational burden significantly (Wucknitz 2010). However, in recent years the advent of "multifield" correlation in VLBI software correlators like DiFX (Deller et al. 2011) have provided an alternative and efficient means of imaging multiple targets within the FoV of the VLBI array. Internal to the correlator, visibilities are processed with high frequency resolution, and are shifted to the positions of the target sources with a high cadence $(\sim 100 \mathrm{~Hz})$ before being heavily averaged (Morgan et al. 2011). In this way hundreds of previously-identified sources can be studied in a single observation at mas resolution, without the generation of an extremely large dataset. 
The main limitation to SKA-VLBI survey science will be the small number of tied-array beams $(\mathrm{N} \sim 4)$ available with SKA1. The relatively sparse nature of the SKA1-MID and SKA1-SUR cores means that only a small fraction of the full primary field of view will be visible to the tied array beams. A larger tied-array beam can be formed at the expense of sensitivity, by including fewer antennas to a shorter maximum diameter. In most cases, however, optimal sensitivity will be gained by using sensitive, narrow tied-array beams and switching between targets with a rapid cadence. Standard multi-field VLBI correlation targeting all sources within the primary beam would be used (since all targets would be visible at all times to the other, single-dish elements of the SKA-VLBI array) but only a subset of the targets would have a tied SKA beam contributing sensitivity at any given time. An important issue is how to calibrate the gain of the remote antennas accurately in the direction of the chosen VLBI phase centres, since the primary beam response of the individual telescopes is often poorly known (Middelberg et al. 2011; Cao et al. 2014; Deller \& Middelberg 2014). This is unlikely to be a concern for tied array beams from SKA1-MID and SKA1-SUR, however, since the primary FoV of the dishes will be order of $1^{\circ}$, considerably larger than some other elements of the SKA-VLBI array (and hence the area from which targets will be drawn).

SKA2 will be capable of streaming and processing data from all individual elements to provide high resolution data for practically the full FoV, although it may still only be practical to provide the full resolution for limited areas, e.g. hundreds of independent phase-centers. The full SKA will thus provide a unique range of mas to arcsec angular scales that will help to distinguish between various interpretations of faint radio sources (unresolved AGN core, resolved emission powered by an AGN, star forming complexes etc.).

\section{Resolving explosive outflows}

SKA-VLBI will make possible (sub)-mas imaging of radio sources. In particular, for transient phenomena such us explosive outflows, SKA-VLBI monitoring will allow us to measure the expansion velocity, or proper motion of jetted ejecta, which is important to understanding the physics of the explosion as well as for studying the progenitor environments. The various Galactic (e.g. microquasars, magnetars, novae) and extragalactic (e.g. TDE) examples are discussed in other chapters. Here we describe a particular application of SKA-VLBI, as an example, in order to highlight the importance of improved sensitivity in global VLBI arrays.

Some of the most energetic events in the universe, supernovae ( $\mathrm{SNe}$ ) and long-duration gammaray bursts, involve explosive outflows of material from dying stars (the latter observed in the other parts of the electromagnetic spectrum as GRB afterglows). Supernovae are not only intrinsically spectacular, but they also have a significant effect on both the chemical evolution of galaxies and inject significant amounts of energy into the interstellar medium. Some supernovae, specifically those of Type Ib and Ic, which originate in stars that have lost most of their original H-rich stellar envelopes, can eject material at relativistic speeds. Long duration GRBs are known to be associated with Type Ic supernovae, and thought to be produced by ultra-relativistic jets launched in the collapsing star. Many details about these processes are still unknown. Why do only a small fraction of Type Ic SNe give rise to an observed GRB? How is the relativistic jet launched and how does it evolve? VLBI provides the best way of obtaining spatially resolved information about these sources, which would be of obvious benefit in attempting to understand the physics. Radio 

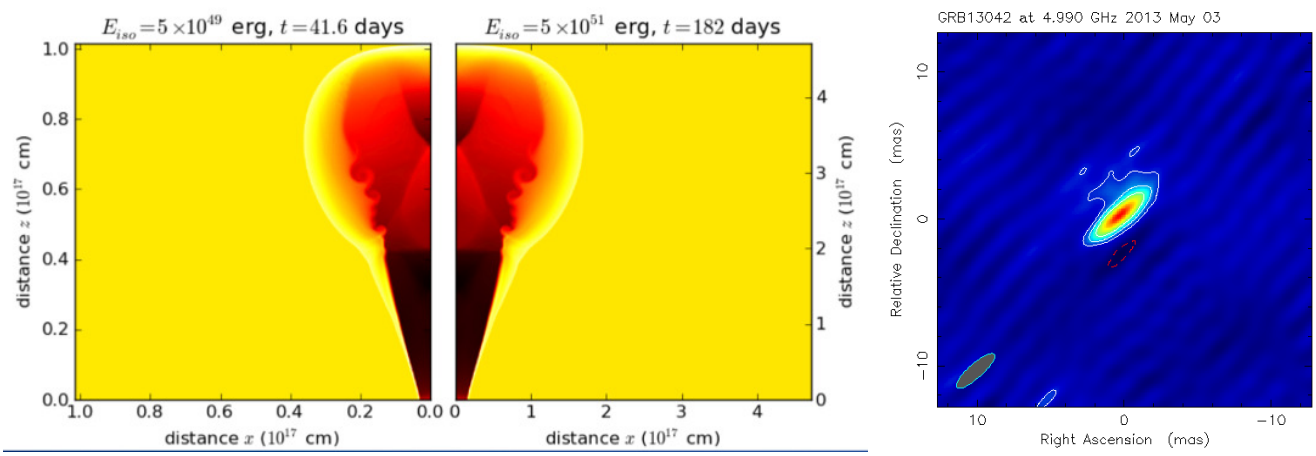

Figure 5: Left: 2D simulations of gamma-ray burst (GRB) jets. The scale invariance between relativistic jets of different energy allows easy reproduction of the jet dynamics. This provides a powerful method for fitting simulation results to observational data (van Eerten et al. 2012). With SKA-VLBI this will be possible for a large number of GRBs. Figure courtesy of Hendrik van Eerten. Right: Early e-EVN detection of the highest fluence GRB detected for decades, GRB 130427A (Paragi et al. 2013).

observations have the further advantage that radio emission generally traces the fastest outflows in GRB afterglows.

VLBI observations of GRBs and SNe are limited both by the available resolution and sensitivity. Although the SKA will provide no direct increase in resolution, it will provide an indirect increase in that the accuracy with which model-fitting can be used to constrain source sizes increases with increasing signal-to-noise ratio (SNR). In SKA1-MID Band 4-5, with a global array of VLBI telescopes where the longest baselines are $\sim 10,000 \mathrm{~km}$ (approximately the distance between the SKA core in South Africa and Australia or Europe), native imaging resolutions down to $\sim 1$ mas (FWHM) would be obtained. For SNR 50, the minimum detectable angular diameter is $\sim 0.2 \mathrm{mas}^{4}$. This minimum detectable size corresponds to a length of $\sim 11$ light-days $\left(3 \times 10^{16} \mathrm{~cm}\right)$ at distance $10 \mathrm{Mpc}$, and $\sim 1.6$ light-years $\left(8 \times 10^{18} \mathrm{~cm}\right)$ at a redshift, $z$ of 0.15 . Interestingly, at $z=20$, it corresponds to the same length of $\sim 1.6$ light years, so sources at very large cosmological distances can be resolved provided they are bright enough to be detected. Such measurements therefore have the exciting potential to directly measure the expansion of a nearby relativistic outflow in the first two weeks, and to possibly resolve outflows even at cosmological distances.

SKA-VLBI will allow us to observe sources below the detection limits of current instruments. Of all GRB afterglows, only $\sim 30 \%$ are bright enough to have been detected in the radio with present instrumentation (Chandra \& Frail 2012), and only GRB 030329 has been resolved (e.g. Taylor et al. 2004). Similarly, only $\sim 30 \%$ of the observed core-collapse supernovae were detected in the radio, and only a handful have well-resolved images (Bietenholz 2008, 2014). The sensitivity of SKA would allow a significantly increased sample of both GRBs and SNe to be resolved with VLBI. We note that the minimum detectable source size could be significantly smaller than 0.1 mas for SNR 1000. This SNR should be achieved for the most extreme and nearby $>1 \mathrm{mJy}$ sources with SKA1, and with SKA2 for practically all GRB afterglows with a flux density of a few hundred $\mu$ Jy known as "radio-loud" today (cf. Table 1). The resulting large GRB samples

\footnotetext{
${ }^{4}$ The theoretical resolving power of an interferometer is inversely proportional to the signal-to-noise ratio. The minimum detectable size depends weakly on the source geometry, and can vary by $~ 30 \%$ for different source geometries such as a uniform disk or an optically thin sphere (Martí-Vidal et al. 2012).
} 
will give the first opportunity to study GRBs in a model-independent fashion while they are still in their ultra-relativistic phase (that can last up to about two weeks for sources that are in a lowdensity environment), and directly compare the results with detailed simulations (see Fig. 5). This will however require extremely accurate calibration for all interferometer elements and flexible response to triggers; detailed simulation of SKA-VLBI configurations will provide more robust estimate of realistic expectations for "super-resolution" capability with SKA-VLBI.

A particularly exciting possibility is that of detecting and resolving the outflows from GRBs from the first generation of stars - Population III GRBs. GRB afterglows have already been detected at $z>9$ (Cucchiara et al. 2011), and recent work (e.g. Ghirlanda et al. 2014; Mesler et al. 2014) has shown that the very massive stars expected to form in the early universe could give rise to spectacular GRBs, and their afterglows may be detectable by SKA despite being at $z \sim 20$.

\section{SKA in VLBI observations: data transport, data formats and correlation}

\subsection{Status of global VLBI}

The EVN currently operates at a bandwidth of $128 \mathrm{MHz}$ and 2-bit sampling, resulting in data streams of $1 \mathrm{Gbit} \mathrm{s}^{-1}$ per telescope. The VLBA in the USA has recently completed an upgrade to $2 \mathrm{Gbit} \mathrm{s}^{-1}$. The ongoing roll-out of new digital backends (DBBC) in the EVN will make 2 and $4 \mathrm{Gbit} \mathrm{s}^{-1}$ operations possible. After the completion of this roll-out, the only obstacles for high-bandwidth global VLBI will be the availability of suitable receiver systems (feeds etc.) at the telescopes, and of sufficient magnetic media for recording or sufficient networking bandwidth for real-time correlation of the data streams. The RFI environment at the different telescope sites will of course limit the usable bandwidth. Using phased-up arrays of radio telescopes (like the ATCA, the VLA and the WSRT) as elements in VLBI observations is common practice. Once beam forming has taken place, the output of the array is treated like that of any of the other VLBI stations.

\subsection{SKA-VLBI}

The cores of SKA1-MID and SKA1-SUR will produce very narrow beams. The practice of using in-beam calibrators in order to obtain the highest quality phase calibration will only be possible if at least four beams are formed, one for the target and additional three for the calibrators (see Sect. 3). With the usual caveats, one can reasonably assume that on the timescale of the construction of SKA1 most VLBI telescopes will move to $512 \mathrm{MHz}$ bandwidth operations. Assuming 4 beams, $512 \mathrm{MHz}$ bandwidth and 2-bit sampling, the total data rate of the phased-up SKA would be $16 \mathrm{Gbit} \mathrm{s}^{-1}$. While current software correlators can easily deal with different bit representations, and a higher bit representation of the SKA data would yield some additional gain in sensitivity, it probably would make most sense to re-sample/truncate (depending on the exact representation) the SKA data at the telescope to 2 bits/sample, thus saving on data volume and storage/transport. The SKA data would also have to conform to the standard VLBI subband scheme, meaning that subbands of $32 \mathrm{MHz}$ should be available. Care should be taken that the band can be tuned so as to be compatible with standard VLBI settings. 


\subsection{Data format}

VLBI has known a variety of data formats. In recent years however a common format named VDIF (Whitney et al. 2009) has been accepted by most of the community. Most newly developed VLBI equipment now supports this data format. While dealing with different formats nowadays is far less problematic than in the days of ASIC-based hardware correlators, one common format is of course preferable. It is not yet known what SKA data will look like, but after beam forming, truncating and maybe re-filtering it should be possible to pack the data in any format desired. Related to this, the SPEAD (Manley et al. 2007) protocol which will be used for data exchange in MeerKAT, and maybe for SKA1-MID as well, actually supports the VDIF format. Hartebeesthoek Observatory in South Africa has participated in a successful $4 \mathrm{Gbit} \mathrm{s}^{-1}$ e-VLBI demonstration, streaming data in real time to the EVN correlator at JIVE in the Netherlands. Considering that $100 \mathrm{Gbit} \mathrm{s}^{-1}$ networking technology is being rolled out by national and international research networks, transporting a data stream of $16 \mathrm{Gbit} \mathrm{s}^{-1}$ from South Africa or Australia to Europe (or another correlator location) in real-time should be no problem at all in five years. Recording such data streams and trickling them to a correlator at lower speeds at a later time ("e-shipping") is already possible right now.

Finally, many VLBI arrays are configured using the VEX (VLBI EXperiment) ${ }^{5}$ format, providing a complete description of a VLBI experiment, including scheduling, data-taking and correlation. Some sort of interface will be needed to translate VEX files into configuration files understandable by the SKA control system.

\section{Conclusions}

In this paper we described the scientific motivation and possible technical realisations of SKAVLBI. The science goals are best achieved with SKA1 by forming phased-array elements from SKA1-MID and SKA1-SUR observing together with existing VLBI arrays in the 1-15 GHz frequency range (and up to $22 \mathrm{GHz}$ in SKA2). In addition, the combination of local interferometry data from SKA1-MID and SKA-VLBI will provide the basis for very accurate amplitude and polarization calibration of the VLBI data products. A high resolution configuration of SKA2 (with a resolution of tens of mas to $\sim 100$ mas, depending on frequency) will allow imaging of e.g. extragalactic sources from sub-pc to kpc scales simultaneously, a capability that is rarely available today. An important part of the requirements for the SKA is forming multiple VLBI beams for accurate phase calibration, targeting multiple sources within the primary beam. This mode of operation will be compatible with other multi-beam VLBI components such as the WSRT-APERTIF.

VLBI with the SKA1 will not be very different from that with other tied arrays, in terms of operations, data rates and computing. More beams will mean more data, but the numbers are reasonable, even in relation to today's technology. As observing at higher frequencies becomes possible with the SKA, increasing the instantaneous bandwidth will become increasingly important. Higher bandwidths, and the consequent higher data rates, should become feasible in the relevant time-frame.

\footnotetext{
${ }^{5}$ http://www.vlbi.org/vex/docs/vex\%20definition\%2015b1.pdf
} 
SKA-VLBI will have a profound effect on a large number of fields within astronomy. Accreting objects will be in reach at a range of accretion rates well below Eddington, providing a comprehensive view of compact objects such as neutron stars as well as the full range of masses from stellar- to supermassive black holes. Many of these will be discovered in transient surveys. Especially interesting candidates are TDEs that could provide clues to derive the low end of the SMBH mass-function, and extremely high redshift GRBs that would provide a line of sight through a large volume of the universe. These, along with the great number of newly discovered dual and multiple SMBH systems and recoiling BH will provide clues for understanding structure formation in the early universe. In addition, there will be a wealth of information about the feedback processes between the central AGN and their host galaxies from spectral line VLBI surveys at $\sim 1 \mathrm{GHz}$. At higher frequencies the study of star formation and stellar evolution, as well as resolving explosive outflows in the Galaxy and at cosmological distances will be among the most important applications. As explained in this work, VLBI astrometry will remain a very important tool for astrophysics. For example pulsar parallax measurements using SKA-VLBI will play an essential role in several high impact areas, including strong field tests of gravity in relativistic binary systems, tomographic mapping of the Galactic magnetic field and mapping the ionised interstellar plasma in the Galaxy, and the physics of neutron stars, as well as detecting the gravitational wave background. A particularly interesting idea is the detection of the gravitational wave background directly through measuring proper motions of a million of quasars with SKA2.

\section{References}

Agudo, I., Böttcher, M., Falcke, H. et al. 2015, "Studies of Relativistic Jets in Active Galactic Nuclei with SKA", in proc. Advancing Astrophysics with the Square Kilometre Array, PoS(AASKA14)093

Asaki, Y., Sudou, H., Kono, Y., et al. 2007, PASJ, 59, 397

Bartel, N., Chandler, J. F., Ratner, M. I., et al. 1996, AJ, 112, 1690

Bietenholz, M. 2008, PoS(IX EVN Symposium)064

Bietenholz, M. F. 2014, PASA, 31, 2

Blandford, R. D., \& Payne, D. G. 1982, MNRAS, 199, 883

Blandford, R. D., \& Znajek, R. L. 1977, MNRAS, 179, 433

Blecha, L., Cox, T. J., Loeb, A., \& Hernquist, L. 2011, MNRAS, 412, 2154

Bourda, G., Charlot, P., \& Le Campion, J.-F. 2008, A\&A, 490, 403

Bourda, G., Charlot, P., Porcas R. W., \& Garrington, S. T. 2010, A\&A, 520, A113

Bower, G. C., Bolatto, A., Ford, E. B., \& Kalas, P. 2009, ApJ, 701, 1922

Burlon, D., Ghirlanda, G., van der Horst, A., et al. 2015, "The SKA view of Gamma-ray Bursts", in proc. Advancing Astrophysics with the Square Kilometre Array, PoS(AASKA14)052

Callegari, S., Kazantzidis, S., Mayer, L. et al. 2011, ApJ, 729, 85

Cao, H.-M., Frey, S., Gurvits, L. I. et al. 2014, A\&A, 563, A111

Chandra, P., \& Frail, D. A. 2012, ApJ, 746, 156

Chi, S., Barthel, P. D., \& Garrett, M. A. 2013, A\&A, 550, A68

Chomiuk, L., Linford, J. D., Yang, J. et al. 2014, Nat, accepted 
Corbel, S., Miller-Jones, J., Fender, R. P. et al. 2015, "Incoherent transient radio emission from stellar-mass compact objects in the SKA era", in proc. Advancing Astrophysics with the Square Kilometre Array, PoS(AASKA14)053

Cucchiara, A., Levan, A. J., Fox, D. B. et al. 2011, ApJ, 736, 7

Deane, R. P., Paragi, Z., Jarvis, M. J. et al. 2014, Nat, 511, 57

Deane, R., Paragi, Z., Jarvis, M., et al. 2015, "Multiple supermassive black holes systems: SKA's future leading role", in proc. Advancing Astrophysics with the Square Kilometre Array, PoS(AASKA14) 151

Deller, A. T., \& Middelberg, E. 2014, AJ, 147, 14

Deller, A. T., Brisken, W. F., Phillips, C. J. et al. 2011, PASP, 123, 275

Deller, A. T., Boyles, J., Lorimer, D. R. et al. 2013, ApJ, 770, 145

Donnarumma, I., Rossi, E. M., Fender, R. P., et al. 2015, "SKA as a powerful hunter of jetted Tidal Disruption Events", in proc. Advancing Astrophysics with the Square Kilometre Array, PoS(AASKA14)054

Fey, A., Boboltz, D., Charlot, P. et al. 2009, Bull. AAS, 41, 676

Fomalont, E., \& Reid, M. 2004, New Astronomy Reviews, 48, 1473

Fomalont, E., Johnston, K., Fey, A. et al. 2011, AJ, 141, 91

Garrett, M. A. 2000, in M. P. van Haarlem (ed.) Perspectives on Radio Astronomy: Science with Large Antenna Arrays, 139 (Astron:Dwingeloo)

Gaylard, M. J., Bietenholz, M. F., Combrinck, L. et al. 2011, in I. Basson \& A. E. Botha (eds.) Proc. SAIP2011, p. 425 (arXiv 1405.7214)

Giroletti, M., Orienti, M., D'Ammando, F. et al. 2015, “The connection between radio and high energy emission in black hole powered systems in the SKA era", in proc. Advancing Astrophysics with the Square Kilometre Array, PoS(AASKA14)153

Gitti, M., Giroletti, M., Giovannini, G., Feretti, L., \& Liuzzo, E. 2013, A\&A, 557, L14

Ghirlanda, G., Burlon, D., Ghisellini, G. 2014, PASA, 31, 22

Godfrey, L., Bignall, H. \& Tingay, S. 2011, "Very High Angular Resolution Science with the SKA", SKA Memo No. 135

Godfrey, L. E. H., Bignall, H., Tingay, S. et al. 2012, PASA, 29, 42

Green, J. A., van Langevelde, H. J., Brunthaler, A. et al. 2015, "Maser Astrometry with VLBI and the SKA", in proc. Advancing Astrophysics with the Square Kilometre Array, PoS(AASKA14) 119

Guirado, J. C., \& Ros, E. 2007, "High-Precision Radio Astrometry: The Search for Extrasolar Planets", in A. Lobanov, A. Zensus, C. Cesarsky \& P. Diamond (eds.) "Exploring the Cosmic Frontier: Astrophysical Instruments for the 21st Century", ESO Astrophysics Symposia, p. 257

Gurvits, L. I. 2004, New Astronomy Reviews, 48, 1211

Gwinn, C. R., Eubanks, T. M., Pyne, T., Birkinshaw, M., \& Matsakis, D. N. 1997, ApJ, 485, 87

Han, J. L., van Straten, W., Lazio, T. J. W., et al. 2015, "Three-dimensional Tomography of the Galactic and Extragalactic Magnetoionic Medium with the SKA" in proc. Advancing Astrophysics with the Square Kilometre Array, PoS(AASKA14)041

Hoffman, L., \& Loeb, A. 2007, MNRAS, 377, 957

Jaffe, A. H. 2004, New Astronomy Reviews, 48, 1482

Janssen, G., Hobbs, G., McLaughlin, M. et al. 2015, "Gravitational wave astronomy with the 
SKA", in proc. Advancing Astrophysics with the Square Kilometre Array, PoS(AASKA14)037

Jimenez-Monferrer, S., Rioja, M. J., Dodson, R., Smirnov, O., \& Guirado, J. C. 2010, PoS(10th EVN Symposium) 084

Komossa, S. 2012, Advances in Astronomy, 2012, id. 364973

Kovalev, Y. Y., Lobanov, A. P., Pushkarev, A. B., \& Zensus, J. A. 2008, A\&A, 483, 759

Kramer, M., \& Stappers, B. 2015, "Pulsar Science with the SKA — Overview", in proc. Advancing Astrophysics with the Square Kilometre Array, PoS(AASKA14)036

Lazio, J. W., Kimball, A., Barger, A. J. et al. 2014, PASP, 126, 196

Lenc, E., Garrett, M. A., Wucknitz, O., Anderson, J. M., \& Tingay, S. J. 2008, ApJ, 673, 78

Lobanov, A. P. 1998, A\&A, 330, 79

Loinard, L., Torres, R. M., Mioduszewski, A. J., et al. 2007, ApJ, 671, 546

Manchester, R. N., Hobbs, G. B., Teoh, A., \& Hobbs, M. 2005, AJ, 129, 1993

Manley, J., Welz, M. Parsons, A., Ratcliffe, S., \& van Rooyen, R. 2007, http://casper.berkeley.edu/astrobaki/images/9/93/ SPEADsignedRelease.pdf

Martínez-Sansigre, A., \& Rawlings, S. 2014, MNRAS, 414, 1937

Martí-Vidal, I., Pérez-Torres, M. A., \& Lobanov, A. P. 2012, A\&A, 541, A135

McKean, J. P., Jackson, N., Vegetti, S. et al. 2015, "Strong gravitational lensing with the SKA", in proc. Advancing Astrophysics with the Square Kilometre Array, PoS(AASKA14)084

Mesler, R. A., Whalen, J., Smidt, J. et al. 2014, ApJ, 787, 91

Middelberg, E., Deller, A., Morgan, J. et al. 2011, A\&A, 526, A74

Mignard, F. 2011, Adv. Sp. Res., 47, 356

Miller-Jones, J. C. A., Jonker, P. G., Dhawan, V., et al. 2009, ApJL, 706, L230

Miller-Jones, J. C. A., Sivakoff, G. R., Knigge, C. et al. 2013, Sci, 340, 950

Morgan, J. S., Mantovani, F., Deller, A. T. et al. 2011, A\&A, 526, A140

Morganti, R., Fogasy, J., Paragi, Z., Oosterloo, T., \& Orienti, M. 2013, Sci, 341, 1082

Morganti, R., Sadler, E. \& Curran, S. J. 2015, "Cool Outflows and HI absorbers with the SKA", in proc. Advancing Astrophysics with the Square Kilometre Array, PoS(AASKA14)134

Nagayama, T., Omodaka, T., Handa, T. et al. 2011, PASJ, 63, 719

Nan, R., Li, Di, Jin, C. et al. 2011, Int. J. Mod. Phys. D, 20, 989

Orosz, G. \& Frey, S. 2013, A\&A, 553, A13

Paragi, Z., Fejes, I., \& Frey, S. 2000, "Indications for Frequency Dependent Radio Core Position in $1823+568$ ", in Nancy R. Vandenberg \& Karen D. Baver (eds.) "IVS 2000 General Meeting", p. 342

Paragi, Z., van der Horst, A. J., Yang, J. et al. 2013, ATel \#5242

Paragi, Z., Frey, S., Kaaret, P. et al. 2014, ApJ, 791, 2

Pen, U.-L., Macquart, J.-P., Deller, A., \& Brisken, W. 2014, MNRAS, 440, L36

Perez-Torres, M. A., Alberdi, A., Beswick, R. J. et al. 2015, "Core-collapse and Type Ia supernoave with the SKA", in proc. Advancing Astrophysics with the Square Kilometre Array, PoS(AASKA14)060

Porcas, R. W. 2012, Mem. Soc. Astron. Ital., 83, 970

Prandoni, I., \& Seymour, N. 2015, "Revealing the Physics and Evolution of Galaxies and Galaxy Clusters with SKA Continuum Surveys" in proc. Advancing Astrophysics with the Square Kilo- 


\section{metre Array, PoS(AASKA14)067}

Ratner, M. I., Bartel, N., Bietenholz, M. F. et al. 2012, ApJS, 201, 5

Reid, M. J., \& Honma, M. 2014, ARA\&A, 52, 339

Reid, M. J., McClintock, J. E., Narayan, R. et al. 2011, ApJ, 742, 83

Rioja, M., Dodson, R., Porcas, R. W. et al. 2009, PoS(EXPReS09)014

Schilizzi, R. T., Alexander, P., Cordes, J. M. et al. 2007, SKA Memo No. 100

Shao, L., Stairs, I. H., Antoniadis, J. et al. 2015, "Testing Gravity with Pulsars in the SKA Era", in proc. Advancing Astrophysics with the Square Kilometre Array, PoS(AASKA14)042

Smits, R., Tingay, S. J., Wex, N., Kramer, M., \& Stappers, B. 2011, A\&A, 528, A108

Taylor, G. B., Frail, D. A., Berger, E., \& Kulkarni, S. V. 2004, ApJ, 609, L1

Titov, O., Lambert, S. B., \& Gontier, A.-M. 2011, A\&A, 529, A91

van Eerten, H., van der Horst, A. J., \& MacFadyen, A. 2012, ApJ, 749, 44

Watts, A. L., Xu, R., Espinoza, C. et al. 2015, "Probing the neutron star interior and the Equation of State of cold dense matter with the SKA", in proc. Advancing Astrophysics with the Square Kilometre Array, PoS(AASKA14)043

Windmark, F., Lindegren, L., \& Hobbs, D. 2011, A\&A, 530, A76

Whitney, A., Kettenis, M., Phillips, C., \& Sekido, M. 2009, PoS(EXPReS09)042.

Wolter, A., Rushton, A. P., Mezcua, M. et al. 2015, "Radio investigation of Ultra-Luminous X-ray (ULX) Sources in the SKA Era", in proc. Advancing Astrophysics with the Square Kilometre Array, PoS(AASKA14)091

Wucknitz, O. 2010, PoS(10th EVN Symposium) 100

Zamaninasab, M., Clausen-Brown, E., Savolainen, T., \& Tchekhovskoy, A. 2014, Nat, 510, 126

Zhang, B., Reid, M. J., Menten, K. M. et al. 2013, ApJ, 775, 79 